\title{
Approximate expressions for modulation speed and threshold for performance optimization of biaxially compressive strain quantum-well lasers
}

\author{
Kerry J. Vahala \\ Department of Applied Physics, Mail Stop 128-95, California Institute of Technology, Pasadena, \\ California 91125 \\ Chung-en Zah \\ Bellcore, 331 Newman Springs Road, Red Bank, New Jersey 07701
}

(Received 10 January 1991; accepted for publication 24 September 1991)

\begin{abstract}
Simple analytical expressions for transparency, threshold, and relaxation oscillation corner frequency are derived for biaxial strain quantum-well lasers. An optimal operating point loss for high speed operation (in the absence of nonlinear gain) is established which varies as the square root of the number of quantum wells. The corresponding relaxation oscillation frequency is found to depend only on fundamental quantities. Its power dependence is $\left[v_{R}(\max )=\left(87 \mathrm{GHz} \sqrt{\mu \mathrm{m}^{3} / \mathrm{mW}}\right)\left(\text { Power }_{\text {out }} / V_{\text {mode }}\right)^{1 / 2}\right]$ where $V_{\text {mode }}$ is the mode volume.
\end{abstract}

Long-wavelength quantum-well lasers employing biaxially compressive-strain active layers based on $\operatorname{In}_{x} \mathrm{Ga}_{1}-{ }_{x}$ As have received much attention recently. Devices fabricated to date have exhibited significantly improved differential quantum efficiencies and low threshold current densities. ${ }^{1-4}$ In addition, theoretical investigations have predicted improved modulation speed. ${ }^{5,6}$ The biaxially strained systems have an unusual band structure. In particular, the highest energy valance band, a heavy-hole band, is well separated in energy from the next highest valence bands. Furthermore, this band has light-hole character for crystal wave vectors in the plane of the quantum well (c.g., the in-plane effective mass is predicted to be 1.5 $m_{l h}$ for typical well widths). ${ }^{7}$ This leads to a nearly symmetrical band structure for the conduction and valance bands involved in lasing. Although this near symmetry is well known, it has not been exploited as a theoretical simplification in studying strained-layer lasers. In this letter, we show that remarkably simple expressions can be developed for transparency, threshold, and modulation response. We compare the predictions of these expressions with numerical results and also use the expressions to consider the problem of modulation response optimization.

The starting point of this analysis is an expression for optical gain in a quantum well system developed by the authors in Ref. 8. For convenience, it is given below,

$$
g(n)=g_{M}\left(1-e^{-n / M N}-e^{-n / M P}\right) .
$$

This expression gives the band edge gain under the assumptions of rigorous $k$-selection and neglect of polarization broadening effects (i.e., $T_{2}=\infty$ ). $n$ is the carrier density in area units for " $M$ ' quantum wells (we assume that the carriers are uniformly distributed among the wells) and $N$ and $P$ are constants which, when necessary, include the effects of filling of higher energy subbands in the conduction and valence bands. General expressions for these constants and the conditions of validity for Eq. (1) are given in Ref. 8. In the present case, the biaxially strained systems, the conditions of validity always hold and $N(P)$ takes on the simple form; $k_{B} T m_{c} / \pi \hbar^{2}\left(k_{B} T m_{v} / \pi \hbar^{2}\right)$ where $m_{c}\left(m_{l}\right)$ is the conduction (valence) band in-plane effective mass. $g_{M}$ is a gain constant given by

$$
g_{M}=\omega|\mu|^{2} m^{*} M / \epsilon \hbar^{2} W,
$$

where $\omega$ is the lasing frequency, $\mu$ is the dipole matrix element, $m^{*}$ is the reduced mass, $M$ is the number of quantum wells, $\epsilon$ is the dielectric constant, and $W$ is the effective width of the optical mode. As discussed in Ref. 8, this expression includes the effect of the incomplete spatial overlap of the optical mode with the quantum wells. $g_{M}$ is, in fact, the maximum attainable modal gain for $M$ quantum wells. Before proceeding, it should also be noted that the effect of doping, as discussed in Ref. 8, is included by making replacements $n / N \rightarrow\left(n+N_{D}\right) / N$ for donors or $n / P \rightarrow\left(n+N_{A}\right) / P$ for acceptors.

Now consider the consequences of a symmetrical band structure. We will make the approximation of perfect symmetry, but test the accuracy of the approximate expressions derived by comparison with plots obtained using Eq. (1) (Note: these plots will sometimes be referred to as "exact" calculations). The starting point is the symmetrical form of Eq. (1).

$$
g(n)=g_{M}\left(1-2 e^{-n / M N}\right) \text {. }
$$

First, consider the transparency condition, which is easily seen to have the form

$$
n_{T}=M N \log 2 .
$$

This expression was, in fact, first derived by Yariv in an analysis of scaling laws in lower-dimensional laser active layers. ${ }^{9}$ It is also possible to find an expression for transparency including the effect of doping (note that $p$-and $n$-type doping are equivalent because of the approximation),

$$
n_{T}=M N \log \left(1+e^{-D / M N}\right) \approx M N e^{-D / M N},
$$

where $D$ is the dopant density in area units for " $M$ " quantum wells and where the approximate form holds for $D$ 


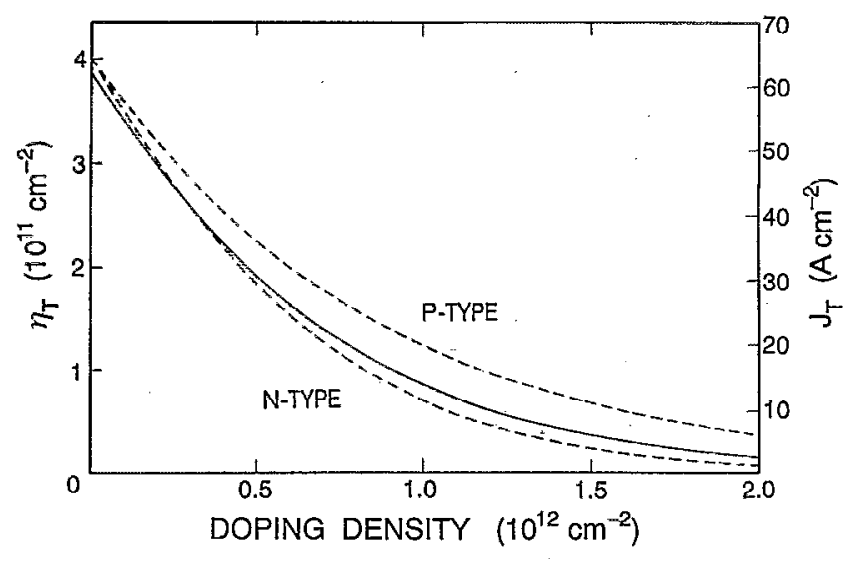

FIG. 1. Transparency carrier density (left-hand axis) and current density (right-hand axis) vs doping density. Solid curve shows the approximate analytical result. Dashed curves are exact calculations for $n$ - and $p$-type doping.

$>M N$. Note that Eq. (4) is recovered for $D=0$. Note that in applying this and other symmetrized expressions, it is necessary to use an effective mass that is, itself, symmetrical in $m_{v}$ and $m_{c}$. We have found that $m=2 m^{*}$ works well and uses this form throughout the analysis. To demonstrate the validity of Eq. (5), we have computed $n_{T}$ versus doping density in Fig. 1 at room temperature for one quantum well. The plot contains three curves. The outer dashed curves result from numerically solving Eq. (1) for the transparency density under the conditions of acceptor and donor doping. The inner solid curve uses the approximation [Eq. (5)]. The agreement is good. Parameters assumed in this calculation and elsewhere in the paper are: $m_{c}=0.04 m_{0}, m_{v}=0.075 m_{0}, W=0.6 \mu \mathrm{m}, \epsilon=12.5 \epsilon_{0}$, and $\mu=(6 \AA) q$, where $\epsilon$ is the dielectric constant of free space and $q$ is the electron charge. For comparison, we have labeled the ordinate in units of carrier density and also current density. For the latter we have assumed a spontaneous lifetime of $1 \mathrm{~ns}$. It is apparent that extremely small transparency currents may be possible with only modest amounts of $p$ - or $n$-type doping.

Threshold in the symmetrical case is also easily obtained by setting $g=1 / \tau$ (note, as mentioned before, the modal confinement is lumped into the definition of $g$ ) where $\tau$ is the photon lifetime. The result is,

$$
n_{T h}=n_{T}+N M \log \left(\frac{\tau g_{M}}{\tau g_{M}-1}\right) \approx n_{T}+\frac{N}{\tau g_{1}},
$$

where the approximate form is valid for $\tau g_{M} \gg 1$ (also note: $\left.g_{M}=M g_{1}\right)$. The unapproximated expression is singular for $1 / \tau=g_{M}$ which, as mentioned before, is the maximum attainable gain for lasing between the lowest energy subbands. In Fig. 2 the threshold carrier density is plotted versus photon lifetime for single and double quantum well active layers. The ordinate, as before, is also labeled in current density units. Note that doping merely shifts these curves downward in accordance with the dependence of transparency density on doping given in Eq. (5). In addition, a set of threshold curves computed by numerically solving Eq. (1) is included for comparison.

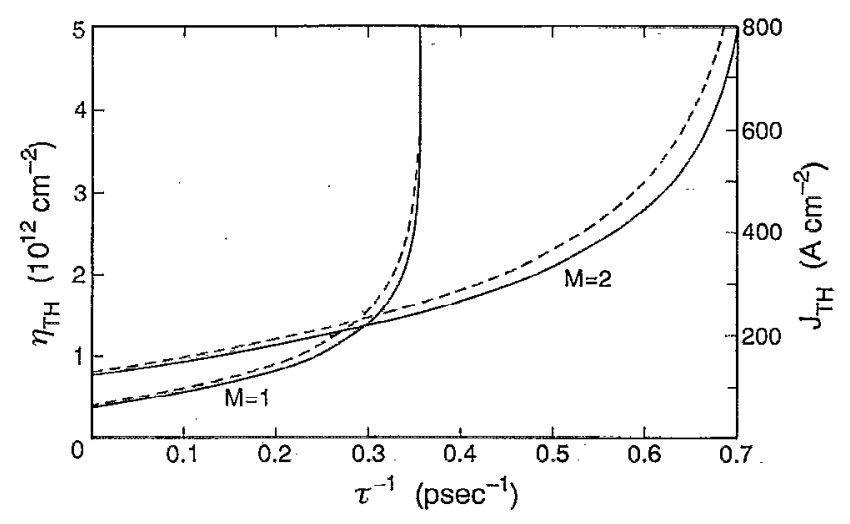

FIG. 2. Approximate (solid) and exact (dashed) threshold carrier density and current density vs cavity loss rate for one quantum well and two quantum well active layers.

Finally, we consider the issue of modulation speed as determined by the relaxation oscillation frequency, which, in units of rad/s, is given by the well known expression

$$
\omega_{R}^{2}=W g g^{\prime} P_{0}=g g^{\prime} S_{0},
$$

where $P_{0}$ and $S_{0}$ are the operating point photon density in volume and area units, respectively (i.e., $S_{0}=W P_{0}$ ), and $g^{\prime}$ is the differential gain (i.e., derivative of modal gain with respect to carrier density in area units). The first form in Eq. (7) has been intentionally written so that both $g$ and $g^{\prime}$ are modal gain quantities (e.g., $W g^{\prime}$ is "material" differential gain ${ }^{5,8}$ ). This will facilitate a substitution made below. The critical material parameters in Eq. (7) are obviously $g g^{\prime}$. It is apparent from Eq. (3) that the quantity $g g^{\prime}$ (and likewise the relaxation oscillation frequency) approaches zero for both low carrier densities (owing to $g$ ) and high carrier densities (owing to $g^{\prime}$ ). (The latter is a qualitative feature that is universal in all quantum well lasers because of subband filling effects.) Owing to the symmetrical approximation, $g^{\prime}$ can be expressed in terms of $g$ which, in turn, can be expressed in terms of cavity loss (i.e., cavity loss $1 / \tau=g$ ). The result is,

$$
\omega_{R}^{2}=\frac{1}{M N}\left(g_{M}-\frac{1}{\tau}\right) \frac{1}{\tau} S_{o}
$$

This expression shows that, beyond operation at high photon densities, speed optimization is determined entirely by modal loss. Maximum speed is attained for $1 / \tau=g_{M} / 2$. This conclusion is independent of doping level since Eq. (8) is easily shown to be valid for any doping density. Equation ( 8 ) has been used in Fig. 3 to plot relaxation oscillation corner frequency versus loss rate for various numbers of quantum wells. $S_{0}=6 \times 10^{10} \mathrm{~cm}^{-2}$ has been assumed (this corresponds to a photon volume density of $10^{15} \mathrm{~cm}^{-3}$ using the modal width $W=0.6 \mu \mathrm{m}$ quoted earlier). For comparison the dashed curves were calculated using a numerical analysis that explicitly includes the effective mass asymmetry. The agreement is good.

In Fig. 3, $\omega_{R}$ increases with increasing cavity loss, but eventually saturates and diminishes with subband-filling induced reduction in the differential gain. The onset of this 


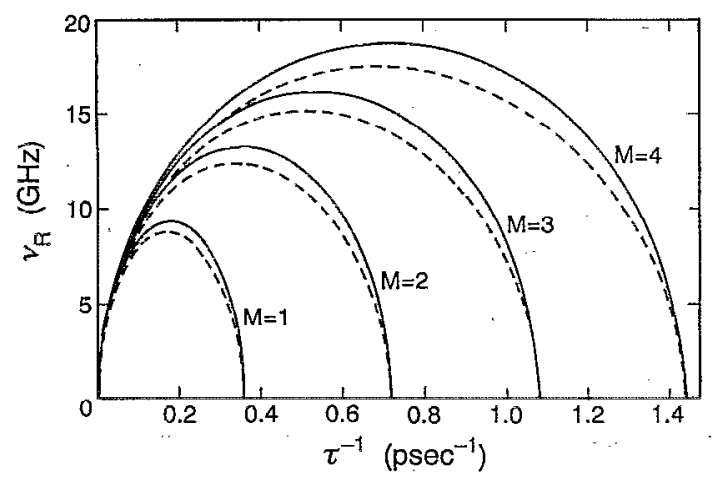

FIG. 3. Relaxation oscillation corner frequency vs cavity loss rate for various numbers of quantum wells (for reference, $1 \mathrm{ps}{ }^{-1}=116 \mathrm{~cm}^{-1}$ ). Both approximate (solid) and exact (dashed) results are shown.

effect occurs at higher loss values for larger numbers of quantum wells. Before continuing we note that the curves in Fig. 3 form a family of ellipses in a normalized modulation-speed/cavity-loss coordinate system. This can be seen upon rearrangement of Eq. (8) which yields $y^{2} / M+4(x-M / 2)^{2} / M^{2}=1$ where $y=\omega_{\mathrm{RM}} / \omega_{R 1}$ and $x=1 / \tau g_{1}$.

We next compute the relaxation oscillation frequency as a function of output power under the condition of optimal loss. For this we will make the simplifying approximation that all losses are facet losses. This is reasonable since typical internal losses average $5 \mathrm{~cm}^{-1}$ per well, which is well below the optimal loss as given by Fig. 3. By letting $1 / \tau=g_{M} / 2$ in Eq. (1) we find

$$
\begin{aligned}
\left.v_{R}\right|_{\max } & =\sqrt{\frac{|\mu|^{2}}{8 \pi k_{B} T \hbar \epsilon}}\left(\frac{\text { Power }_{\text {out }}}{V_{\text {mode }}}\right)^{1 / 2} \\
& =\left(87 \mathrm{GHz} \sqrt{\frac{\mu \mathrm{m}^{3}}{\mathrm{~mW}}}\right)\left(\frac{\text { Power }_{\text {out }}}{V_{\text {mode }}}\right)^{1 / 2},
\end{aligned}
$$

where $V_{\text {mode }}$ is the mode volume. The coefficient in this expression depends only on material or thermodynamic quantities. The dependence on quantum well number appearing in Fig. 3 disappears here owing to selection of optimum loss and calculation in terms of output power. Under these conditions output power is proportional to $M g_{1} S_{0}$ so that, for example, a four quantum well device would have four times lower photon density than a one quantum well device at the same output power. Hence, from Fig. 3, each device would have the same speed versus power relation as indicated by Eq. (9). We note, however, that in practice the bandwidth could be limited by damping at high photon densities due to the nonlinear gain effect.

Finally, we note that the optimal loss condition $1 / \tau=g=g_{M} / 2$ can be substituted into Eq. (6) to arrive at the following threshold carrier density (undoped layer) for optimum speed

$$
\left.n_{\text {Th }}\right|_{\text {optimum speed }}=2 n_{T} \text {. }
$$

That is, the highest corner frequency results when the threshold carrier density is simply twice the transparency carrier density. For a doped active layer, the optimum carrier density is simply $\left(n_{T}\right)_{\text {undoped }}+\left(n_{T}\right)_{\text {doped }}$ in accordance with Eqs. (5) and (6).

In conclusion, we have shown that extremely simple, analytical expressions can be derived for transparency carrier density, threshold carrier density, and relaxation oscillation corner frequency in the biaxial strain quantum well systems. The approximation utilizes the near-symmetrical structure of the lowest lying conduction and valence bands in these systems. The dependence of transparency current density on doping density and threshold current density on loss were investigated. In addition an expression giving the relaxation oscillation corner frequency in terms of only the cavity loss (as opposed to loss and differential gain) was derived, allowing an optimum operating point loss for high speed operation in the absence of nonlinear gain to be established. At a fixed photon density, the maximum relaxation oscillation frequency scales as the square root of the number of quantum wells and the optimum loss scales linearly with the number of wells. The power dependence of the maximum relaxation oscillation frequency is a simple analytical expression that depends only on temperature, dipole strength, and modal volume.

The authors would like to thank T. P. Lee for several useful discussions and comments.

'P. J. A. Thijs and T. Van Dongen, Electron. Lett. 25, 1735 (1989).

${ }^{2}$ U. Koren, M. Oron, M. G. Young, B. I. Miller, J. L. DeMiguel, G. Raybon, and M. Chien, Electron. Lett. 26, 465 (1990).

${ }^{3}$ C. E. Zah, F. J. Favire, R. Bhat, S. G. Menocal, N. C. Andreadakis, D. M. Hwang, M. Kuza, and T. P. Lee, Tech. Digest, 12th IEEE Int. Semiconductor Laser Conf., Davos, Switzerland, 42-43 (1990).

${ }^{4}$ T. Tanbun-Ek; R. A. Logan, N. A. Olsson, H. Temkin, A. M. Sargent, and $\mathrm{K}$. W. Wecht, Tech. Digest, 12th IEEE Int. Semiconductor Laser Conf., Davos, Switzerland, 46-47 (1990).

${ }^{5}$ K. Y. Lau, S. Xin, W. I. Wang, N. Bar-Chaim, and M. Mittelstein, Appl. Phys. Lett. 55, 1173 (1989).

${ }^{6}$ I. Suemune, L. A. Coldren, M. Yamanishi, and Y. Kan, Appl. Phys. Lett. 53, 1378 (1988).

${ }^{7}$ M. Houng and Y. C. Chang, J. Appl. Phys. 65, 3096 (1989).

${ }^{8}$ K. Vahala and C. E. Zah, Appl. Phys. Lett. 52, 1945 (1988).

${ }^{\circ}$ A. Yariv, Appl. Phys. Lett. 53, 1033 (19.88). 